\title{
Long-term effects of recurrent intermittent hypoxia and hyperoxia on respiratory system mechanics in neonatal mice
}

\author{
Andrew M. Dylag' ', Catherine A. Mayer', Thomas M. Raffay', Richard J. Martin', Anjum Jafri' and Peter M. MacFarlane'
}

BACKGROUND: Premature infants are at increased risk for wheezing disorders. Clinically, these neonates experience recurrent episodes of apnea and desaturation often treated by increasing the fraction of inspired oxygen $\left(\mathrm{FIO}_{2}\right)$. We developed a novel paradigm of neonatal intermittent hypoxia with subsequent hyperoxia overshoots $\left(\mathrm{ClH}_{\mathrm{O} / \mathrm{E}}\right)$ and hypothesized that $\mathrm{ClH}_{\mathrm{O} / \mathrm{E}}$ elicits long-term changes on pulmonary mechanics in mice.t

METHODS: Neonatal C57BL/6 mice received $\mathrm{ClH}_{\mathrm{O} / \mathrm{E}^{\prime}}$ which consisted of $10 \% \mathrm{O} 2(1 \mathrm{~min})$ followed by a transient exposure to $50 \% \mathrm{FIO}_{2}$, on 10 -min repeating cycles $24 \mathrm{~h} / \mathrm{d}$ from birth to P7. Baseline respiratory mechanics, methacholine challenge, RT-PCR for pro and antioxidants, radial alveolar counts, and airway smooth muscle actin were assessed at P21 after 2-wk room air recovery. Control groups were mice exposed to normoxia, chronic intermittent hyperoxia $\left(\mathrm{ClH}_{\mathrm{E}}\right)$, and chronic intermittent hypoxia $\left(\mathrm{ClH}_{0}\right)$.

RESULTS: $\mathrm{ClH}_{\mathrm{O} / \mathrm{E}}$ and $\mathrm{ClH}_{\mathrm{E}}$ increased airway resistance at higher doses of methacholine and decreased baseline compliance compared with normoxia mice. Lung mRNA for NOX2 was increased by $\mathrm{ClH}_{\mathrm{O} / \mathrm{E}}$. Radial alveolar counts and airway smooth muscle actin was not different between groups.

CONCLUSION: Neonatal intermittent hypoxia/hyperoxia exposure results in long-term changes in respiratory mechanics. We speculate that recurrent desaturation with hyperoxia overshoot may increase oxidative stress and contribute to wheezing in former preterm infants.

B ronchopulmonary Dysplasia (BPD) is the major pulmonary morbidity of prematurity, affecting up to 10,000 US infants annually (1). Increased airway reactivity and wheezing disorders are among the most common long-term pulmonary consequences of prematurity and BPD and worsen with increasing degree of prematurity $(2,3)$. Studies of former preterm infants demonstrate a combination of increased airway resistance, decreased lung compliance, and/or increased health care utilization (hospitalizations and asthma medications) from term corrected age through adulthood (4-8). Physicians in the Neonatal Intensive Care Unit (NICU) are challenged with maintaining adequate levels of $\mathrm{O}_{2}$ saturation while avoiding excessive periods of hypoxia and hyperoxia. Recurrent apnea and respiratory insufficiency associated with apnea of prematurity lead to frequent episodes of $\mathrm{O}_{2}$ desaturation despite supplemental $\mathrm{O}_{2}$ therapy (9). Recent studies in preterm infants using pulse oximetry have well characterized the timing and incidence of these events in the first 2 mo of postnatal life (10). The incidence of desaturations $(<80 \%$ saturation for 10-180 s) increases dramatically during the first two postnatal weeks followed by a steady decline thereafter with extremely preterm infants experiencing over 100 desaturation events per day (11). In the clinical setting, these events are often resolved by a transient increase in the fraction of inspired oxygen $\left(\mathrm{FIO}_{2}\right)$; most commonly titrated by the bedside nurse. A consequence of increasing $\mathrm{FIO}_{2}$ to resolve the desaturation event, however, is an ensuing "overshoot" and a transient period of relative hyperoxia above baseline (12). Clinical studies demonstrate that the majority of desaturation events treated with $\mathrm{O}_{2}$ result in higher $\mathrm{O}_{2}$ saturations compared with an infant's target range (13). Furthermore, there is often a period of several minutes where the delivered $\mathrm{FIO}_{2}$ is greater than baseline after the event resolves (13). These episodes of reoxygenation following an $\mathrm{O}_{2}$ desaturation event may be expected to exacerbate oxidative stress, but their effects on long term airway reactivity and wheezing disorders in premature infants are unknown.

Murine lung development at birth is in the saccular stage of alveolarization, similar to the premature human lung at 24-36 wk gestation, making it a suitable candidate to model lung disease secondary to prematurity (14). This model has been validated in numerous animal models of BPD which frequently utilize sustained and continuous hyperoxia exposure to replicate the BPD phenotype (15). Animal models of chronic intermittent hypoxia $\left(\mathrm{CIH}_{\mathrm{O}}\right)$ have similarly been shown to worsen allergen-induced pulmonary inflammation and have deleterious effects on respiratory function (16). $\mathrm{CIH}_{\mathrm{O}}$ is also associated with increased levels of NADPH oxidases (NOX)2 and reactive oxygen species (ROS) production (17). Furthermore, reoxygenation with $50 \% \mathrm{FIO}_{2}$ after acute hypoxia exposure resulted in a transient surge in superoxide anion formation (18). This would suggest that hypoxia

'Department of Pediatrics, Division of Neonatology, Rainbow Babies and Children's Hospital, Case Western Reserve University School of Medicine, Cleveland, Ohio. Correspondence: Peter M. MacFarlane (pmaca@case.edu)

Received 17 May 2016; accepted 19 September 2016; advance online publication 1 March 2017. doi:10.1038/pr.2016.240 


\section{Articles $\mid$ Dylagetal.}

followed by reoxygenation with hyperoxia may be a potent stimulus of ROS formation. Despite these findings, the specific patterns that predispose to later lung airway injury have not been well characterized. In this study, we developed a novel neonatal mouse model of $\mathrm{CIH}_{\mathrm{O}}$ exposure with hyperoxia overshoots to determine if they have a deleterious effect on subsequent airway hyper-reactivity and respiratory mechanics. We hypothesized that neonatal $\mathrm{CIH}_{\mathrm{O}}$ with superimposed intermittent hyperoxia causes an increase in airway responsiveness to methacholine challenge and is associated with changes in prooxidant enzymes.

\section{METHODS}

\section{Animals and Exposures}

Animal protocols were approved by the Institutional Animal Care and Use Committee at Case Western Reserve University. Timed pregnant C57BL/6 female mice (Jackson Laboratories, Bar Harbor, ME) were purchased and housed in an AAALAC accredited animal facility on 12-h light-dark cycles with ad libitum standard food and water. Newborn mouse pups were pooled within $24 \mathrm{~h}$ of birth and randomly redistributed into treatment groups. Each litter and the nursing dam were placed in a modified exposure chamber designed to permit rapid exchange of chamber gas composition according to the desired exposure paradigm. Gas flow into the chamber was modified using individual mass flow controllers (SDPROC, AALBORG Instruments \& Controls, Orangeburg, NY), programmed with a command module and corresponding software. Each flow control was independently controlled to allow airflow of gases (air, nitrogen or oxygen) into the chamber to achieve the desired exposure paradigm. An $\mathrm{O}_{2}$ sensor was placed into the exposure chamber to continuously record achieved $\mathrm{FIO}_{2}$. A schematic of the experimental setup is shown in Figure 1.

To simulate a desaturation with hyperoxia overshoot, one group of mice were exposed to repeating cycles of hypoxia $\left(10 \% \mathrm{O}_{2}\right)$ immediately followed by rebound hyperoxia $\left(50 \% \mathrm{O}_{2}\right)$. After the chamber reached $50 \% \mathrm{O}_{2}$, room air ( $21 \%$ oxygen) was purged into the chamber allowing gradual return of chamber conditions to normoxia $\left(\mathrm{N}_{\mathrm{x}}\right)$ within $5 \mathrm{~min}$ (Figure 1b). This exposure was repeated at $10 \mathrm{~min}$ intervals, $24 \mathrm{~h} / \mathrm{d}$ for $7 \mathrm{~d}$ starting at P0. This treatment group that received intermittent hypoxia and hyperoxia overshoot was designated $\mathrm{CIH}_{\mathrm{O} / \mathrm{E}}$. Additional groups of mice were used to assess the separate effects of $\mathrm{CIH}_{\mathrm{O}}$ or hyperoxia $\left(\mathrm{CIH}_{\mathrm{f}}\right)$. All groups were compared with control mice raised in room air for the entire exposure period. After $7 \mathrm{~d}$ of respective exposures, animals were returned to room air until respiratory function testing or tissue harvesting at P21.

\section{Respiratory Mechanics}

Respiratory function testing was performed at P21 for all groups ( $n=7-11$ per group). Each animal was placed under general anesthesia (intraperitoneal ketamine $200 \mathrm{mg} / \mathrm{kg}$ and xylazine $20 \mathrm{mg} / \mathrm{kg}$ ), tracheostomized via a 19-gauge blunt tip cannula, and placed on a commercially available small mammal ventilator (flexiVent, SCIREQ, Montreal, Canada). Each subject was then paralyzed (pancuronium bromide $10 \mathrm{mg} / \mathrm{kg}$ ) and mechanically ventilated with a tidal volume of $10 \mathrm{ml} / \mathrm{kg}, 150$ breaths/min, PEEP of $3 \mathrm{~cm} \mathrm{H}_{2} \mathrm{O}$, and $\mathrm{FIO}_{2}$ of $50 \%$. Animals equilibrated for $5 \mathrm{~min}$ on the ventilator, then two recruitment breaths of sustained inspiration up to a pressure of $30 \mathrm{~cm} \mathrm{H}_{2} \mathrm{O}$

a

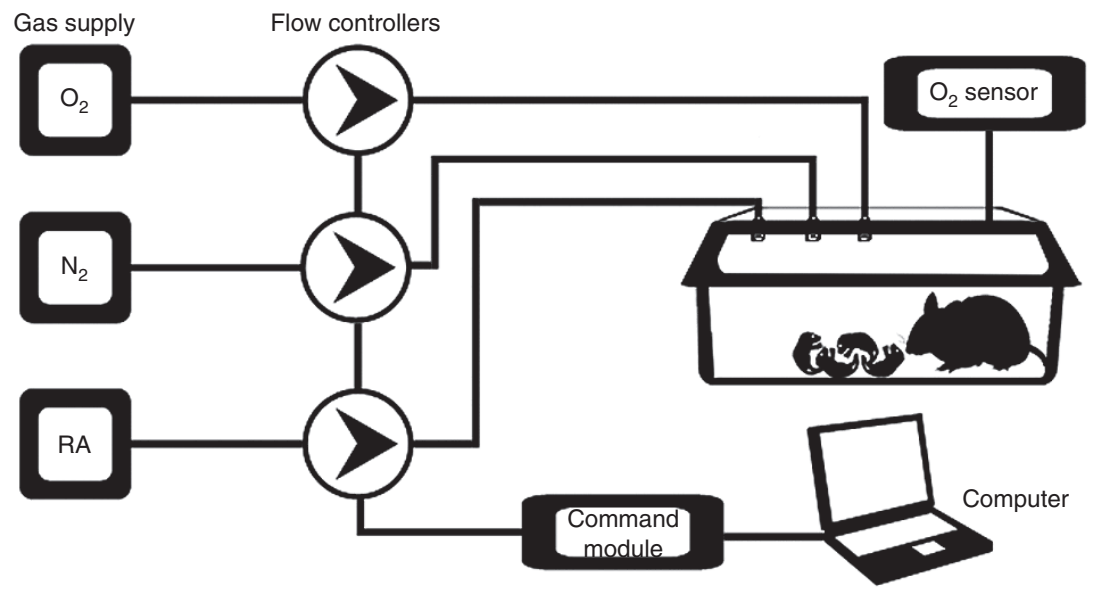

b

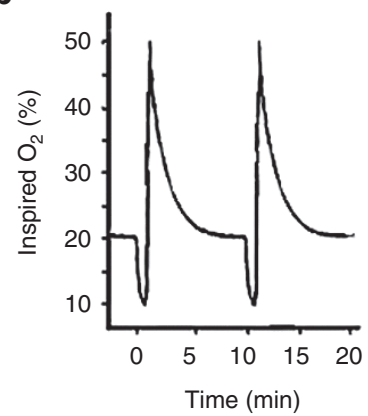

Chronic intermittent

Hypoxia/Hyperoxia $\left(\mathrm{ClH}_{\mathrm{O} / \mathrm{E}}\right)$
C

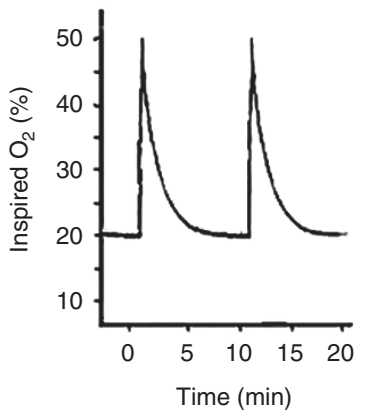

Chronic intermittent Hyperoxia $\left(\mathrm{ClH}_{\mathrm{E}}\right)$ d

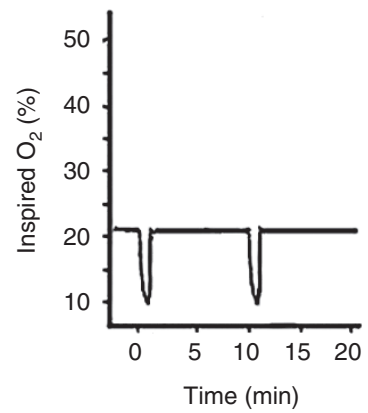

Chronic intermittent Hypoxia $\left(\mathrm{ClH}_{\mathrm{O}}\right)$

Figure 1. Intermittent hypoxia and hyperoxia exposure system. (a) Schematic of experimental setup. Independent gas inputs of Nitrogen ( $\left.\mathrm{N}_{2}\right)$, Oxygen $\left(\mathrm{O}_{2}\right)$ and room air (RA) pass through computer-programmed flow controllers each connected to a command module and operated by a computer. Gas inputs feed into the animal cage attached to oximeter. Oxygen exposure paradigm for each experimental group with continuously monitored levels: (b) Chronic intermittent hypoxia/hyperoxia (c) Chronic intermittent hyperoxia (d) Chronic intermittent hypoxia. Room air controls not illustrated. Two 10-min repeating cycles illustrated. Cycles repeat $24 \mathrm{~h} / \mathrm{d}$. 
for $3 \mathrm{~s}$ were administered followed by measurement of baseline respiratory mechanics. A dose-response curve was generated by administering aerosolized (Aeroneb, SCIREQ) methacholine (Sigma Aldrich, St. Louis, MO) for $10 \mathrm{~s}$ at increasing doses (saline control, 12.5, 25, 50,100 , and $200 \mathrm{mg} / \mathrm{ml}$ ). Respiratory system resistance (Rrs) and specific respiratory system compliance (sCrs) were calculated by the ventilator software (Flexiware Version 7.2 Service Pack 2 Build 728, SCIREQ, Montreal, Canada) using a $1.2 \mathrm{~s}, 2.5 \mathrm{~Hz}$ single-frequency forced oscillation maneuver (19). Specific tissue damping (sG) and specific elastance $(\mathrm{sH})$ were calculated using a low-frequency forced oscillation technique imparting a quasi-sinusoidal maneuver containing a wide range of frequencies. Input impedance was then analyzed through the previously described Constant Phase Model (20). Specific values for respiratory function parameters were determined by dividing the result by the inspiratory capacity of each animal at baseline.

\section{Real Time PCR}

RNA was extracted from snap-frozen lungs of $21 \mathrm{~d}$ old mice $(n=$ 7-16 per group) using TriZol reagent (Life Technologies, Carlsbad, CA) and quantified by Nanodrop spectroscopy (Thermo Scientific, Waltham, MA). cDNA was generated from $1 \mu \mathrm{g}$ of RNA by reverse transcription using qScript cDNA synthesis kit (Quanta Biosciences, Gaithersburg, MD). Real-time quantitative PCR was performed on a StepOne PCR system (Applied Biosystems, Foster City, CA) using TaqMan probes (Life Technologies) for superoxide dismutases (SOD)1 (Mm01344233_g1), SOD2 (Mm01313000_m1), SOD3 (Mm01213380_s1), NOX1 (Mm00549170_m1), NOX2 (Mm01287743_m1), NRF2 (Mm00801891_m1) compared with glyceraldehyde 3-phosphate dehydrogenase (GAPDH) (\#4351309) with PerfeCTa quantitative PCR FastMix, UNG, ROX (Quanta Biosciences, Beverly, MA). Fold-changes are reported utilizing $2^{\wedge}$-ddCT method and StepOne Software v2.3 (Applied Biosystems).

\section{Histology and Morphometric Analysis}

Animals ( $n=7-12$ per group) were sacrificed using anesthetic overdose of ketamine/xylazine and lungs inflated with $4 \%$ paraformaldehyde at $25 \mathrm{~cm} \mathrm{H_{2 }} \mathrm{O}$ pressure on postnatal day 21. Paraffin-embedded sections $(5 \mu \mathrm{m})$ were prepared and stained with hematoxylin-eosin. Randomly chosen hematoxylin-eosin areas were photographed with $10 \times$ magnification from 7-10 animals per group. All of the morphometric and image analysis measurements were performed by a blinded investigator. The extent of alveolarization was determined by the radial alveolar counts (RAC) method as previously described (21). Six terminal bronchioles per pup were analyzed for RAC, determined by counting the number of alveolar septa transected by a perpendicular line drawn from the terminal bronchiole to the nearest connective tissue septum.

\section{Immunofluorescence and Analysis for Airway Smooth Muscle Actin}

The trachea was cannulated and the lungs ( $n=8-10$ per group) were inflation fixed $\left(25 \mathrm{~cm} \mathrm{H}_{2} \mathrm{O}\right)$ for 10 min with $4 \%$ neutral-buffered formalin. The left lung was removed, prepared for immuno-staining, and postfixed for $2 \mathrm{~d}$ at $4^{\circ} \mathrm{C}$. The fixed lobe was dehydrated in graded alcohol and embedded in paraffin. Deparaffinized tissue sections were pretreated for antigen retrieval, and incubated with a monoclonal antibody against mouse anti $\alpha$-smooth muscle actin ( $\alpha$-ASM; 1:400 dilution, Sigma-Aldrich, St. Louis, MO) overnight at $4^{\circ} \mathrm{C}$. Immune complexes were captured with FITC-conjugated donkey antimouse (1:500, Alexa Fluor-488, Invitrogen, Carlsbad, CA). Appropriate negative controls were run by omitting the primary antibody to confirm nonspecific staining, which was not observed. Immunostained sections were cover slipped with Vectashield mounting medium (Vector Laboratories, Burlingame, CA) and visualized with a fluorescence microscope. Images of the immunostained sections were captured with a Rolera XR CCD camera (Q-Imaging, Surrey, Canada) mounted on a microscope (Leica Microsystems, Wetzlar, Germany). Five random images at $20 \times$ magnifications were captured from five to seven animals per group by using digital image analysis software with settings for color and size identification (Image $j$, NIH, Bethesda, MD).
The area of airways and the green fluorescent areas $\alpha$-ASM from five airways per animal were measured using the circular method (22). The amount of $\alpha$-ASM area was expressed as $\alpha$-ASM staining divided by airway basement membrane perimeter $\left(P_{\mathrm{bm}}\right)$.

\section{Statistical Analysis}

Data are expressed as mean \pm SEM with exception of animal weights (mean \pm SD). Respiratory mechanics at baseline and for each dose of methacholine exposure were performed using two-way ANOVA with repeated measures. Analysis of mRNA expression was performed with a one-way ANOVA on the $\triangle \mathrm{CT}$. RAC and alpha smooth muscle
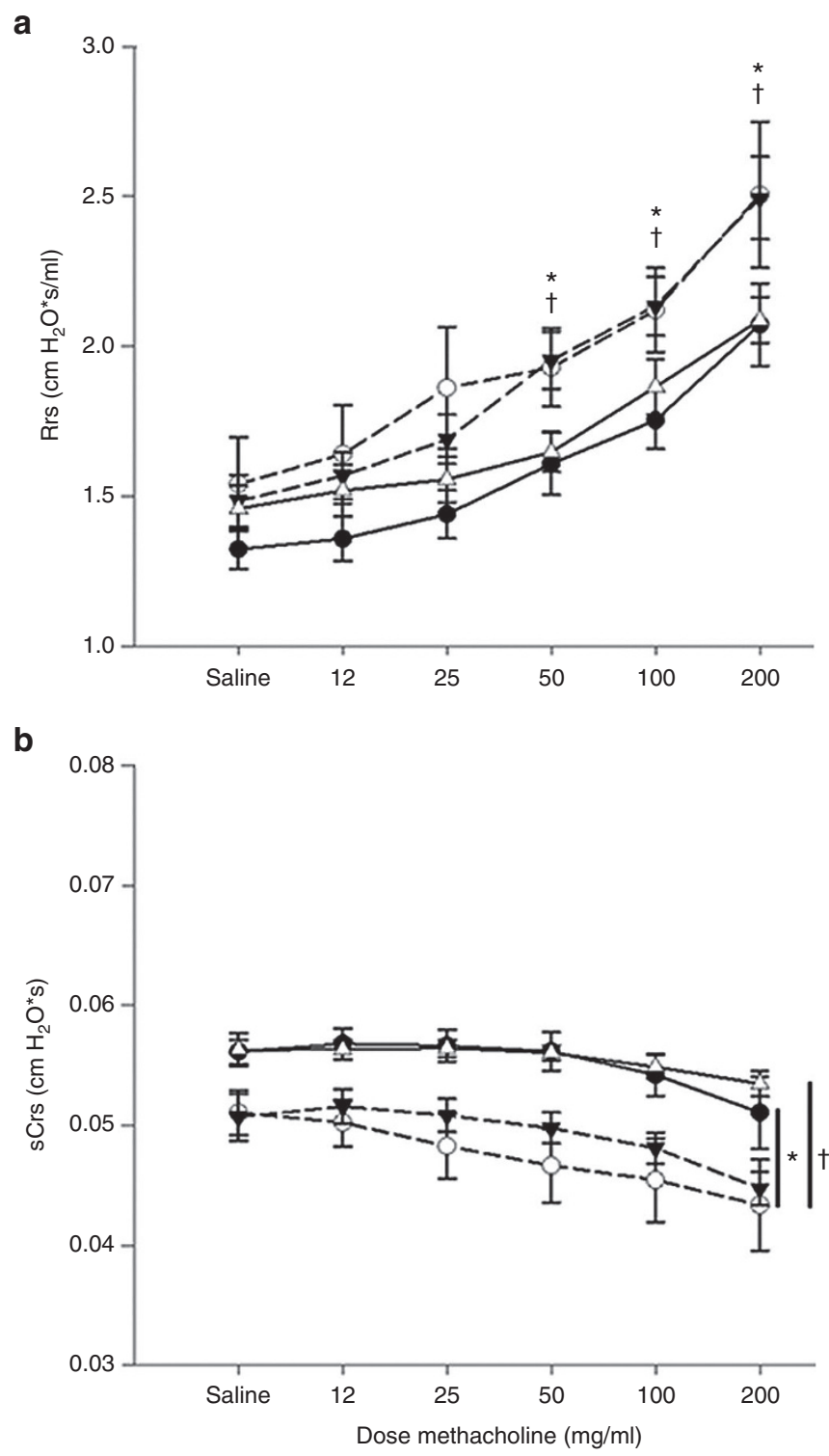

Figure 2. Respiratory system resistance (Rrs) and specific respiratory system compliance (sCrs) and responses to methacholine in mechanically ventilated mice at P21. (a) Significant increases in Rrs were observed at higher methacholine doses of both groups who received intermittent hyperoxia $\left(\mathrm{ClH}_{\mathrm{O} / \mathrm{E}^{\prime}}\right.$ dashed line closed triangles and $\mathrm{CIH}_{\mathrm{E}^{\prime}}$ dashed line open circles) compared with room air controls ( $\mathrm{N}_{x^{\prime}}$ solid line closed circles). (b) Significant decrease in $\mathrm{sCrs}$ in $\mathrm{ClH}_{\mathrm{O} / \mathrm{E}}$ and $\mathrm{ClH}_{\mathrm{E}}$ at all doses compared with room air controls. No changes were observed between $\mathrm{ClH}_{0}$ (solid line, open triangles) and $\mathrm{N}_{\mathrm{X}} \cdot n=7-11$ per group. $P<0.05$ : ${ }^{*} \mathrm{CIH}_{\mathrm{O} / \mathrm{E}}$ Vs. $\mathrm{N}_{\mathrm{X}^{\prime}}$ ${ }^{+} \mathrm{ClH}_{\mathrm{E}}$ vs. $\mathrm{N}_{\mathrm{x}}$. 


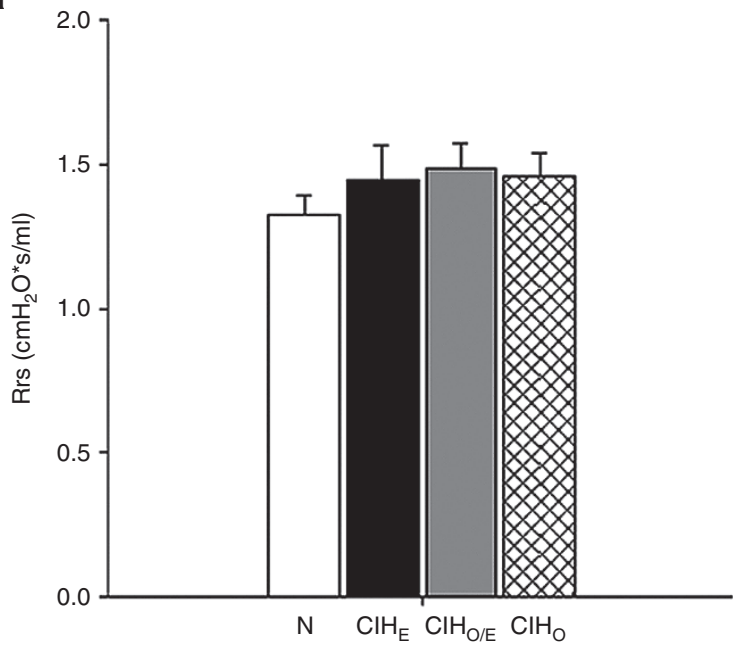

C

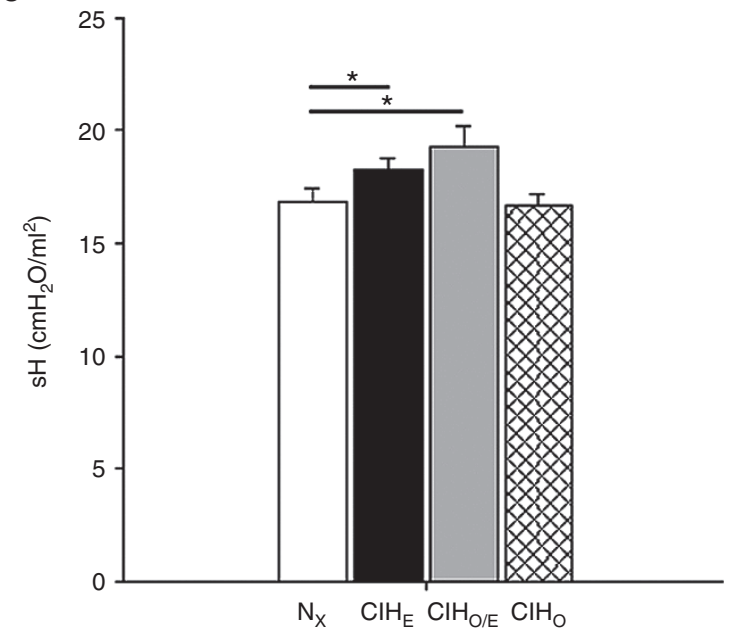

b

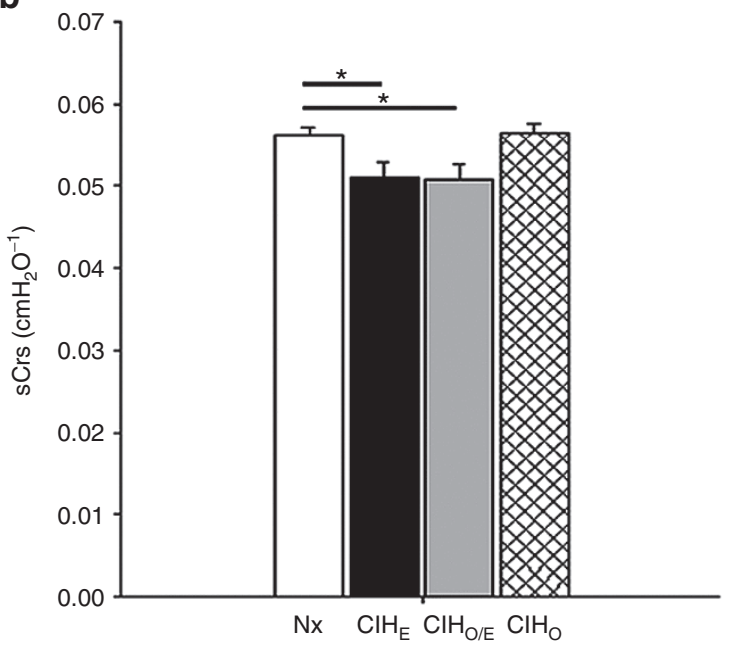

d

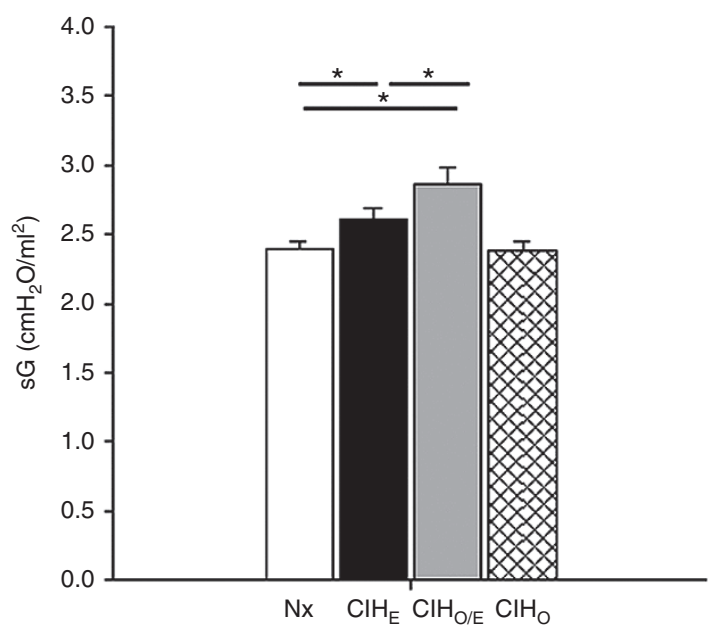

Figure 3. Baseline respiratory mechanics at P21. (a) Respiratory system resistance (Rrs) (b) Specific respiratory system compliance (sCrs) (c) Specific elastance (sH). (d) Specific tissue damping (sG). $n=7-11$ per group. ${ }^{*} P<0.05$.

actin were performed with one-way ANOVA. Statistical significance was defined as $P<0.05$.

\section{RESULTS}

\section{Body Weight}

Average body weight was not significantly different between groups at $\mathrm{P} 21\left(\mathrm{~N}_{\mathrm{X}}: 8.9 \pm 1.9 \mathrm{~g}_{\mathrm{CIH}}: 8.1 \pm 1.5 \mathrm{~g} ; \mathrm{CIH}_{\mathrm{O} / \mathrm{E}}: 8.4 \pm 1.4 \mathrm{~g}\right.$; and $\left.\mathrm{CIH}_{\mathrm{O}}: 8.4 \pm 1.9 \mathrm{~g} ; P=0.25\right)$.

\section{Airway Responsiveness to Methacholine}

Within each group there was a significant dose-dependent increase in Rrs (Figure 2a) and decrease in Crs (Figure 2b) when challenged with methacholine. $\mathrm{CIH}_{\mathrm{O} / \mathrm{E}}$ and $\mathrm{CIH}_{\mathrm{E}}$ groups demonstrated increased Rrs at higher methacholine doses (50$200 \mathrm{mg} / \mathrm{ml}$ ) compared with animals raised in room air (Figure 2a; $P<0.05)$. Airway reactivity, defined as the difference between maximal contraction at the highest dose of methacholine and baseline, did not increase in the $\mathrm{CIH}_{\mathrm{O} / \mathrm{E}}$ or $\mathrm{CIH}_{\mathrm{E}}$ groups compared with $\mathrm{N}_{\mathrm{X}}(P=0.15$ and $P=0.53$ respectively). There was a consistent decrease in sCrs at all methacholine doses among the $\mathrm{CIH}_{\mathrm{O} / \mathrm{E}}$ and $\mathrm{CIH}_{\mathrm{E}}$ groups compared with $\mathrm{N}_{\mathrm{X}}$ (Figure $2 \mathrm{~b} ; P<0.05$ ).
There were no differences in resistance or compliance responses observed in the $\mathrm{CIH}_{\mathrm{O}}$ group compared with room air controls.

\section{Baseline Respiratory Mechanics}

Baseline respiratory mechanics at P21 are shown in Figure 3. No differences in Rrs were observed between groups (Figure 3a) at baseline. Baseline sCrs was decreased and specific elastance $(\mathrm{sH})$ increased in the $\mathrm{CIH}_{\mathrm{O} / \mathrm{E}}$ and $\mathrm{CIH}_{\mathrm{E}}$ groups compared with room air controls (both $P<0.05$; Figure $3 \mathbf{b}, \mathbf{c}$ ). Similarly, sG was increased in the $\mathrm{CIH}_{\mathrm{O} / \mathrm{E}}$ and $\mathrm{CIH}_{\mathrm{E}}$ groups compared with room air controls $(P<0.05$, Figure $3 \mathrm{~d})$. The $\mathrm{CIH}_{\mathrm{O} / \mathrm{E}}$ group demonstrated a further increase in sG compared with the $\mathrm{CIH}_{\mathrm{E}}$ subjects $(P<0.05$, Figure $3 d)$. No significant baseline differences were seen in the $\mathrm{CIH}_{\mathrm{O}}$ group compared with room air controls.

\section{Real Time PCR}

In an effort to describe changes in pro-oxidants and antioxidants gene expression, real time PCR of lung homogenates was performed at P21 for various SOD and NOX. The antioxidants SOD1, SOD2, and SOD3 and their relative fold-change from 


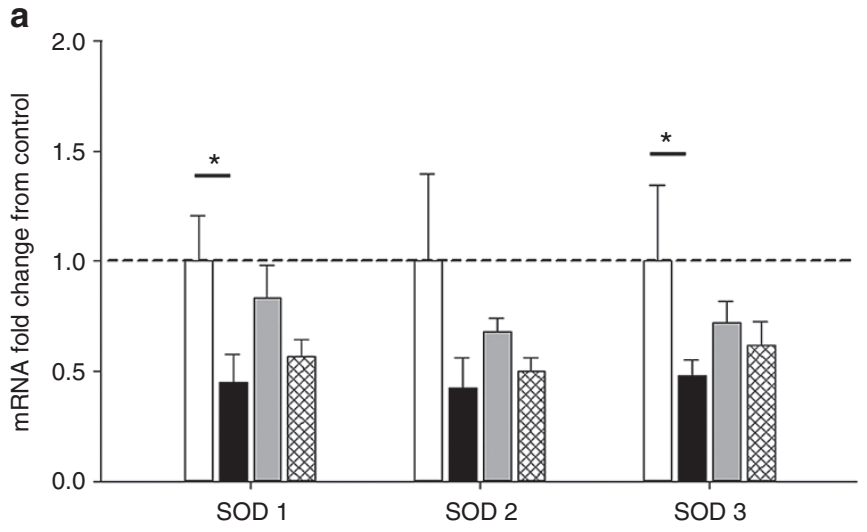

b

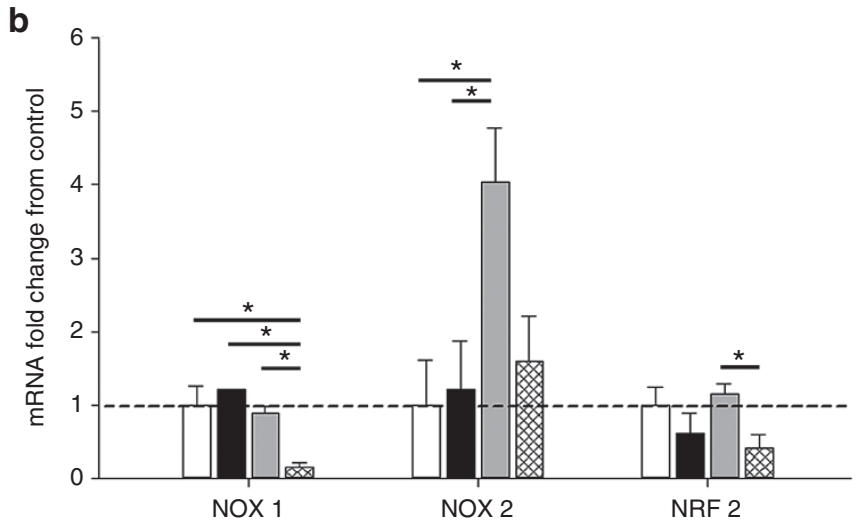

Figure 4. Real-time PCR of lung homogenates. RNA was extracted from whole lung homogenates of pups at P21. Fold-change of mRNA expression are demonstrated for groups chronic intermittent hyperoxia $\left(\mathrm{ClH}_{\mathrm{E}}\right)$ (second from left, black), $\mathrm{ClH}_{\mathrm{O} / \mathrm{E}}$ (third from left, gray) and chronic intermittent hypoxia $\left(\mathrm{ClH}_{\mathrm{o}}\right)$ (right, hatched) normalized to $\mathrm{Nx}$ (left, white) mice.

(a) Antioxidant markers superoxide dismutases (SOD) 1, SOD2, and SOD3 (b) Pro-oxidant makers of NADPH oxidases (NOX)1, NOX2, and NRF2. $n=$ 7-16 per group. ${ }^{*} P<0.05$.

$\mathrm{N}_{\mathrm{X}}$ are shown (Figure 4a). SOD1 and SOD3 were decreased in $\mathrm{CIH}_{\mathrm{E}}$ animals compared with $\mathrm{N}_{\mathrm{X}}$ but unchanged among other groups. SOD2 was unchanged in all groups. The pro-oxidants NOX1, NOX2, as well as the transcription factor and antioxidant-regulating protein nuclear factor (erythroid-derived-2)like2 (NRF2) were also evaluated (Figure $4 \mathrm{~b}$ ). NOX1 was decreased in $\mathrm{CIH}_{\mathrm{O}}$ compared with all other groups $(P<0.05)$. NOX2 was increased in $\mathrm{CIH}_{\mathrm{O} / \mathrm{E}}$ compared with all other groups $(P<0.05)$. NRF2 was decreased in $\mathrm{CIH}_{\mathrm{O}}$ compared with $\mathrm{CIH}_{\mathrm{O} / \mathrm{E}}$.

\section{Immunohistochemistry}

To assess long-term effects of intermittent hypoxia and hyperoxia on alveolarization, we quantified RAC at P21 (Figure 5). There were no significant differences in RAC between groups. There were also no differences in the amount of airway $\alpha$-smooth muscle actin between treatment groups. Representative images and staining for $\alpha$-smooth muscle actin are demonstrated in Figure 6.

\section{DISCUSSION}

Prematurity is an independent risk factor for wheezing disorders among extremely and moderately preterm infants,

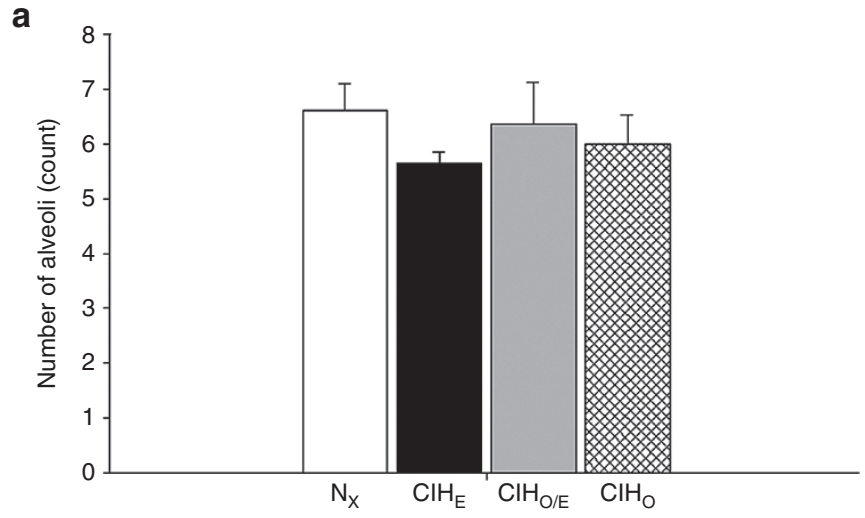

b

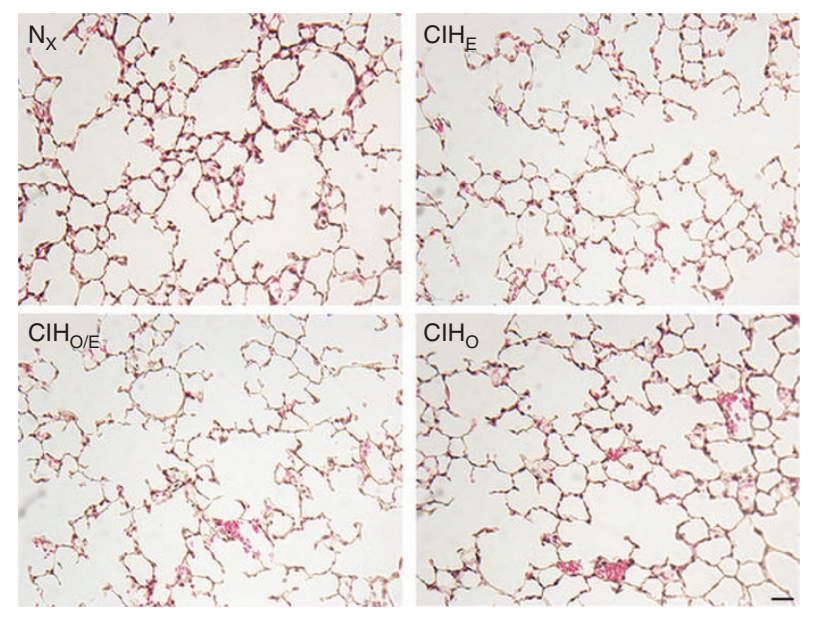

Figure 5. Radial alveolar counts for all groups at P21. (a) Graph of counts for all four groups (b) Representative images of each group. No differences were seen between groups. Magnification bar $50 \mu \mathrm{m} . n=7-12$ per group.

attributed to structural lung immaturity, pulmonary surfactant deficiency, mechanical ventilation, and supplemental oxygen therapy (23). Furthermore, emerging evidence implicates even late preterm birth as a risk factor for impaired respiratory function attributable to differences in lung and chest wall compliance, airway tethering, and susceptibility to postnatal infections such as RSV (24). Among infants born preterm, small airway obstruction is evident with increased odds of childhood asthma and decreased forced expiratory volume among infants with and without $\operatorname{BPD}(25,26)$. We pursued this study to determine if a mouse model of $\mathrm{FIO}_{2}$ fluctuations and delivery of specific patterns of oxygen exposure, contribute to pulmonary morbidity by altering respiratory mechanics, lung architecture, ASM, and changes in pro-oxidant and antioxidant signaling.

In these experiments, $\mathrm{CIH}_{\mathrm{O} / \mathrm{E}}$ increased airway resistance at higher methacholine doses, decreased compliance, increased elastance, and increased tissue damping in pulmonary function testing. sG characterizes energy dissipation in the lung and markedly increases when there are regional differences in lung ventilation (20). sG increased in $\mathrm{CIH}_{\mathrm{O} / \mathrm{E}}$ compared with $\mathrm{CIH}_{\mathrm{E}}$ suggesting that hypoxia immediately followed by hyperoxia may have some synergistic negative effect on regional respiratory mechanics. $\mathrm{CIH}_{\mathrm{O}}$ alone had no effect on pulmonary mechanics, lung architecture, or ASM compared with 


\section{Articles Dylagetal.}

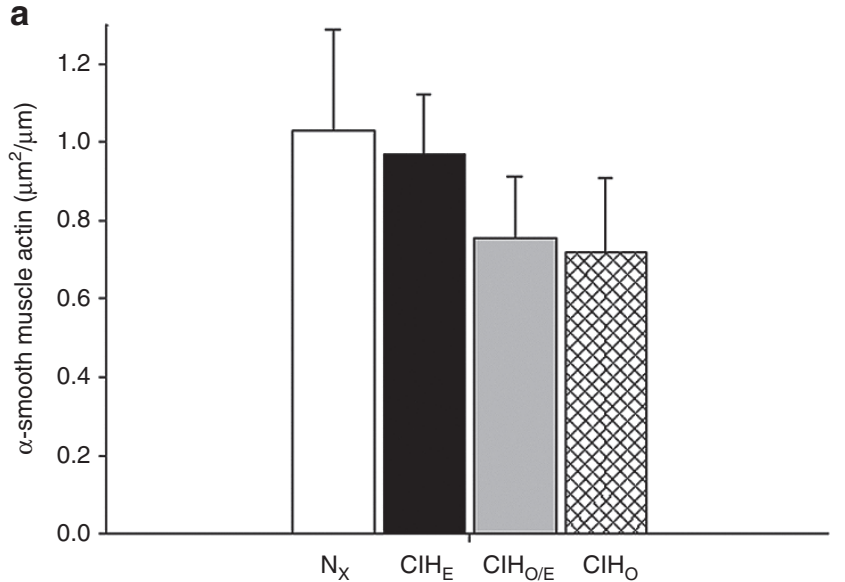

b
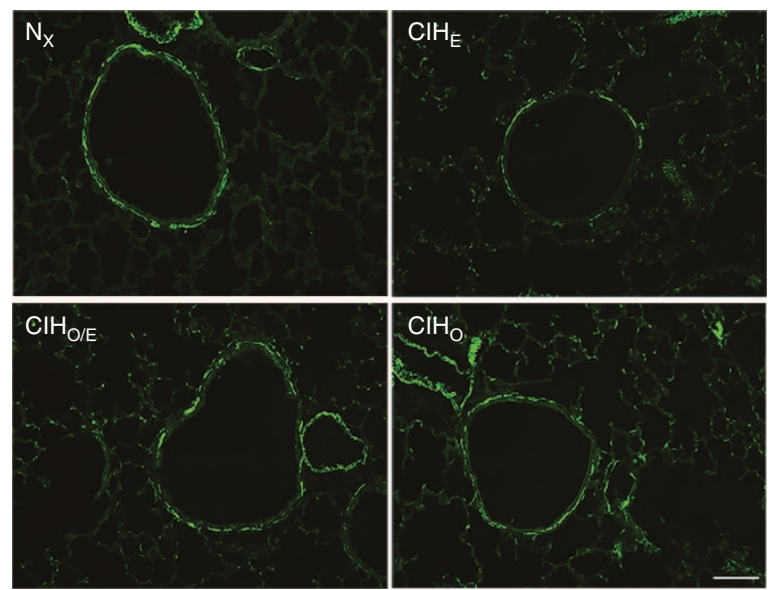

Figure 6. Alpha-actin in airway smooth muscle (ASM) at P21. (a) Graph of alpha-smooth muscle actin expression in all four groups and (b) representative images from each group. No significant differences were observed between groups. Magnification bar $50 \mu \mathrm{m} . n=8-10$ per group.

$\mathrm{N}_{\mathrm{x}}$ controls. We did not, however, observe any corresponding changes in RAC, ASM hypertrophy, and mRNA expression for antioxidant and pro-oxidant markers except NOX2 in our $\mathrm{CIH}_{\mathrm{O} / \mathrm{E}}$ treated mice. Animals exposed to $\mathrm{CIH}_{\mathrm{E}}$ had changes almost identical to $\mathrm{CIH}_{\mathrm{O} / \mathrm{E}}$ with the exception that increased NOX2 mRNA was unique to the latter group. Although these data demonstrate complex changes in lung mRNA for various pro and antioxidant enzymes, the most significant observation was the increased Rrs to methacholine in the $\mathrm{CIH}_{\mathrm{O} / \mathrm{E}}$ group was associated with an increase in mRNA for the pro-oxidant NOX2. Taken together, these findings suggest that min-to-min swings in $\mathrm{FIO}_{2}$ above and below $21 \%$ are relevant stimuli that contribute to altered pulmonary mechanics.

The nature of intermittent hypoxia and hyperoxia events in preterm infants changes with advancing postnatal age in both timing and frequency (11). Altering these patterns places different stressors on the developing airway, potentially altering signaling pathways and disrupting lung development. Our study utilized a pattern of exposure with $\mathrm{FIO}_{2}$ cycling between 10 and $50 \%$ in $10-\mathrm{min}$ intervals for $24 \mathrm{~h} / \mathrm{d}$, differentiating this exposure paradigm from other conventional exposures of sustained continuous hyperoxia that have shown airway hyper-reactivity, ASM hypertrophy, and decreased bronchiolar-alveolar attachments $(27,28)$. In addition, our model addresses the intermittent hypoxia/hyperoxia fluctuations that take place in neonatal intensive care units on the order of minutes, not hours and mimics the frequency of events (up to $100 / \mathrm{d}$ ) documented in the NICU (11). We demonstrated deleterious effects on pulmonary function with an average $\mathrm{FIO}_{2}$, calculated as the "area under the curve", of $30-32 \%$ in the $\mathrm{CIH}_{\mathrm{O} / \mathrm{E}}$ group. These physiologic changes persist at P21, roughly correlating to the pulmonary stage in development for a toddler-aged child (14), a common point where respiratory morbidity is described from chronic lung disease due to prematurity (29). $\mathrm{CIH}_{\mathrm{O}}$ in the absence of hyperoxia overshoot did not induce long term airway morbidity. Therefore, our characterization of at-risk patterns of intermittent hypoxia and hyperoxia may aid in guiding bedside management of labile preterm infants experiencing frequent apnea and desaturations to reduce hyperoxia overshoots and potentially their long-term risk of pulmonary morbidity.

Studies with different timing, frequency, and severity of intermittent hypoxia/hyperoxia compared with $\mathrm{CIH}_{\mathrm{O} / \mathrm{E}}$ have observed dysfunction in angiogenic and alveolar signaling pathways. One mouse model with single, 2-h cycles of hypoxia (12\%) with hyperoxia (50\%) for $7 \mathrm{~d}$ demonstrated downregulation of HIF- $1 \alpha$ and VEGF, implicating altered angiogenesis and thus impaired alveolar development (30). A different paradigm of clustered intermittent hypoxia superimposed on sustained hyperoxia showed alterations in VEGF levels normalized by treatment with a superoxide dismutase mimetic, further implicating altered angiogenesis as a factor (31). It is clear that the amount, severity, timing, and pattern of these swings in oxygen exposure can have wide-ranging effects on several signaling pathways, making it an intriguing area for further study.

The mechanism by which intermittent hypoxia with hyperoxia contributes to deleterious lung function is still not well established. In contrast to previously published studies of intermittent hypoxia/hyperoxia at $1 \mathrm{wk}$ (30), alveolarization was not affected in any study group following room air recovery at P21. We proceeded to investigate changes in signaling pathways that might be implicated in functional changes in the lung. RT-PCR for both pro-oxidant and antioxidant markers demonstrated increased NOX2 in only the $\mathrm{CIH}_{\mathrm{O} / \mathrm{E}}$ group. NOX2 was the first protein in the family of NADPH oxidase proteins to be discovered and is present in many different cell types with highest expression in phagocytes (32). It produces superoxide, peroxynitrites, and other ROS that are involved in host defense (32). Counts for alveolar macrophages (33) were not different between the $\mathrm{CIH}_{\mathrm{O} / \mathrm{E}}$ group and other groups (data not shown), suggesting the increase in NOX2 signaling is independent of the presence of inflammatory cells in the lung parenchyma. Further investigation is needed to investigate what role NOX2 is playing in this $\mathrm{CIH}_{\mathrm{O} / \mathrm{E}}$ paradigm and how it interacts with other physiologic stressors.

We have evaluated changes in both ASM and lung parenchyma as both may contribute to increased airway reactivity. The absence of ASM hypertrophy is consistent with no change 
in baseline Rrs and decreased compliance might be associated with decreased alveolarization although we did not detect changes in alveolar counts. Therefore, we speculate that the pattern of intermittent exposures is having an effect on lung function that may not be anatomically detectable at this time point. These findings contrast with other models of sustained hyperoxia demonstrating increased ASM hypertrophy with $40 \%$ sustained $\mathrm{FIO}_{2}$ (28) underscoring the importance of studying fluctuations in inspired $\mathrm{FIO}_{2}$ as they relate to pulmonary function and airway reactivity.

In conclusion, the $\mathrm{CIH}_{\mathrm{O} / \mathrm{E}}$ paradigm of intermittent hypoxia with hyperoxia overshoots for $7 \mathrm{~d}$ has long-term deleterious effects on lung function at P21 after 2-wk recovery in room air but no detectable effect on alveolarization or smooth muscle hypertrophy. This study, like others underscores the negative effect specific patterns of oxygen exposure can have on lung development and lung function. Piecing together the cumulative dose of oxygen with the pattern in which it is delivered may provide valuable insight into how we respond to these events at the bedside. Increased oxidative stress may be implicated, however, the intermittent nature of these exposures may elicit a different signature of oxygen toxicity compared with models of sustained hyperoxia. These experiments are a first step in characterizing one pattern of oxygen exposure that may be implicated in long-term respiratory morbidity in preterm infants.

\section{STATEMENT OF FINANCIAL SUPPORT}

This work was supported by the American Academy of Pediatrics Marshal Klaus Perinatal Research Award (Elk Grove Village, IL) and an endowment from the Rainbow Babies and Children's Foundation Fellowship Research Award Program (Cleveland, $\mathrm{OH}$ ). Additional support provided by the National Institutes of Health T32-HD060537 training grant (Bethesda, MD). Dylag is the recipient of the National Institutes of Health Loan Repayment Program award (Bethesda, MD).

Disclosure: The authors report no conflict of interest.

\section{REFERENCES}

1. Jobe AH, Bancalari E. Bronchopulmonary dysplasia. Am J Respir Crit Care Med 2001;163:1723-9.

2. Baraldi E, Carraro S, Filippone M. Bronchopulmonary dysplasia: definitions and long-term respiratory outcome. Early Hum Dev 2009;85(10 Suppl):S1-3.

3. Ehrenkranz RA, Walsh MC, Vohr BR, et al.; National Institutes of Child Health and Human Development Neonatal Research Network. Validation of the National Institutes of Health consensus definition of bronchopulmonary dysplasia. Pediatrics 2005;116:1353-60.

4. Hjalmarson O, Sandberg K. Abnormal lung function in healthy preterm infants. Am J Respir Crit Care Med 2002;165:83-7.

5. Pramana IA, Latzin P, Schlapbach LJ, et al. Respiratory symptoms in preterm infants: burden of disease in the first year of life. Eur J Med Res 2011;16:223-30.

6. Hennessy EM, Bracewell MA, Wood N, et al.; EPICure Study Group. Respiratory health in pre-school and school age children following extremely preterm birth. Arch Dis Child 2008;93:1037-43.

7. Fawke J, Lum S, Kirkby J, et al. Lung function and respiratory symptoms at 11 years in children born extremely preterm: the EPICure study. Am J Respir Crit Care Med 2010;182:237-45.

8. Northway WH Jr, Moss RB, Carlisle KB, et al. Late pulmonary sequelae of bronchopulmonary dysplasia. N Engl J Med 1990;323:1793-9.

9. Abu-Shaweesh JM, Martin RJ. Neonatal apnea: what's new? Pediatr Pulmonol 2008;43:937-44.

10. Martin RJ, Wang K, Köroğlu O, Di Fiore J, Kc P. Intermittent hypoxic episodes in preterm infants: do they matter? Neonatology 2011;100:303-10.
11. Di Fiore JM, Bloom JN, Orge F, et al. A higher incidence of intermittent hypoxemic episodes is associated with severe retinopathy of prematurity. J Pediatr 2010;157:69-73.

12. Baerts W, Lemmers PM, van Bel F. Cerebral oxygenation and oxygen extraction in the preterm infant during desaturation: effects of increasing $\mathrm{FiO}(2)$ to assist recovery. Neonatology 2011;99:65-72.

13. van Zanten HA, Tan RN, Thio M, et al. The risk for hyperoxaemia after apnoea, bradycardia and hypoxaemia in preterm infants. Archives of disease in childhood Fetal and Neonatal Edition 2014;99:F269-73.

14. Amy RW, Bowes D, Burri PH, Haines J, Thurlbeck WM. Postnatal growth of the mouse lung. J Anat 1977;124(Pt 1):131-51.

15. Hilgendorff A, Reiss I, Ehrhardt H, Eickelberg O, Alvira CM. Chronic lung disease in the preterm infant. Lessons learned from animal models. Am J Respir Cell Mol Biol 2014;50:233-45.

16. Broytman O, Braun RK, Morgan BJ, et al. Effects of chronic intermittent hypoxia on allergen-induced airway inflammation in rats. Am J Respir Cell Mol Biol 2015;52:162-70.

17. Peng YJ, Nanduri J, Yuan G, et al. NADPH oxidase is required for the sensory plasticity of the carotid body by chronic intermittent hypoxia. J Neurosci 2009;29:4903-10.

18. Fabian RH, Perez-Polo JR, Kent TA. Extracellular superoxide concentration increases following cerebral hypoxia but does not affect cerebral blood flow. Int J Dev Neurosci 2004;22:225-30.

19. Shalaby KH, Gold LG, Schuessler TF, Martin JG, Robichaud A. Combined forced oscillation and forced expiration measurements in mice for the assessment of airway hyperresponsiveness. Respir Res 2010;11:82.

20. Hantos Z, Daróczy B, Suki B, Nagy S, Fredberg JJ. Input impedance and peripheral inhomogeneity of dog lungs. J Appl Physiol (1985) 1992;72: 168-78.

21. Emery JL, Mithal A. The number of alveoli in the terminal respiratory unit of man during late intrauterine life and childhood. Arch Dis Child 1960;35:544-7.

22. O’Reilly M, Hansbro PM, Horvat JC, Beckett EL, Harding R, Sozo F. Bronchiolar remodeling in adult mice following neonatal exposure to hyperoxia: relation to growth. Anat Rec (Hoboken) 2014;297:758-69.

23. Been JV, Lugtenberg MJ, Smets E, et al. Preterm birth and childhood wheezing disorders: a systematic review and meta-analysis. PLoS Med 2014;11:e1001596.

24. Colin AA, McEvoy C, Castile RG. Respiratory morbidity and lung function in preterm infants of 32 to 36 weeks' gestational age. Pediatrics 2010;126:115-28.

25. Kotecha SJ, Edwards MO, Watkins WJ, et al. Effect of preterm birth on later FEV1: a systematic review and meta-analysis. Thorax 2013;68:760-6.

26. Jaakkola JJ, Ahmed P, Ieromnimon A, et al. Preterm delivery and asthma: a systematic review and meta-analysis. J Allergy Clin Immunol 2006;118:823-30.

27. O’Reilly M, Harding R, Sozo F. Altered small airways in aged mice following neonatal exposure to hyperoxic gas. Neonatology 2014;105: $39-45$.

28. Wang H, Jafri A, Martin RJ, et al. Severity of neonatal hyperoxia determines structural and functional changes in developing mouse airway. Am J Physiol Lung Cell Mol Physiol 2014;307:L295-301.

29. Stevens TP, Finer NN, Carlo WA, et al.; SUPPORT Study Group of the Eunice Kennedy Shriver National Institute of Child Health and Human Development Neonatal Research Network. Respiratory outcomes of the surfactant positive pressure and oximetry randomized trial (SUPPORT). J Pediatr 2014;165:240-249.e4.

30. Elberson VD, Nielsen LC, Wang H, Kumar HS. Effects of intermittent hypoxia and hyperoxia on angiogenesis and lung development in newborn mice. J Neonatal Perinatal Med 2015;8:313-22.

31. Chang M, Bany-Mohammed F, Kenney MC, Beharry KD. Effects of a superoxide dismutase mimetic on biomarkers of lung angiogenesis and alveolarization during hyperoxia with intermittent hypoxia. Am J Transl Res 2013;5:594-607.

32. Bedard K, Krause KH. The NOX family of ROS-generating NADPH oxidases: physiology and pathophysiology. Physiol Rev 2007;87: 245-313.

33. Reyburn B, Di Fiore JM, Raffay T, et al. The effect of continuous positive airway pressure in a mouse model of hyperoxic neonatal lung injury. Neonatology 2016;109:6-13. 